\title{
Phenotypic detection of Extended Spectrum Beta- Lactamase producing uropathogens using DDST, PCT, Chrom agar and E-test - A comparitive study
}

\author{
R. Prabha*, Joshy M. Easow and M. Swapna \\ Veeramamunivar street, Radhakrishnan nagar, Pondicherry 605009, India \\ *Corresponding author
}

\author{
A B S T R A C T
}

\begin{tabular}{l} 
K e y w o r d s \\
ESBL; Extended \\
Spectrum Beta \\
Lactamase, DDST - \\
Double Disc Synergy \\
Test, PCT; \\
Phenotypic \\
Confirmatory Test, \\
E-test - Epsilomer \\
test. \\
\hline Article Info \\
\hline Accepted: \\
19 March 2016 \\
Available Online: \\
10 April 2016
\end{tabular}

Urinary tract infections (UTI) are a serious threat to human health affecting millions of people every year resulting in high morbidity and mortality. Enterobacteriaceae species like Escherichia coli, Proteus mirabilis, Enterobacter aerogenes, Citrobacter freundii and Klebsiella pneumoniae are the commonest uropathogens implicated in bacteremia and nosocomial infections. Aims and objectives: To identify and study the incidence of extended spectrum beta-lactamase producing Enterobacteriaceae in urinary tract infections by different phenotypic detection tests. About 100 Uropathogens isolated from urine samples of inpatients and outpatients of SVMCH during 2014-2015 were included. ESBL producing isolates were identified by screening methods and confirmed by phenotypic methods like DDST, PCT, E-test and CHROM agar. Among 52 isolates of E. coli 40\% was ESBL producer, among 4 isolates of Citrobacter 50\% was ESBL producer, out of 5 isolates of P. vulgaris 20\% was ESBL producer, out of 6 isolates of K. oxytoca 33\% was ESBL producer, out of 13 isolates of P. mirabilis $23 \%$ was ESBL producer, out of 16 isolates of $\mathrm{K}$. pneumoniae $25 \%$ was ESBL producer, out of 3 isolates of E. aerogenes $67 \%$ was ESBL producer and none of the isolates of $M$. morgagnii was ESBL producer. By DDST, $29 \%$ of ESBL producing isolates and $71 \%$ of non-ESBL isolates were detected. With PCT, E-test and ESBL CHROM agar 35\% of ESBL producers and 65\% of non-ESBL isolates were detected. Conclusion: For early detection of ESBL producers, methods like DDST, PCT can be performed in microbiology laboratories as routine test, as they are simple to perform and cost effective. Those isolates which are found to be ESBL producers by screening methods can be confirmed by tests like E-test, ESBL CHROM agar which are found to be equally effective in detecting ESBL producers.

\section{Introduction}

Urinary tract infections (UTI), also known as acute cystitis or bladder infection are a serious threat to human health affecting millions of people every year resulting in high morbidity and mortality. The presence and growth of microorganisms anywhere in the urinary tract is the UTI and it is the commonest bacterial infection of mankind ${ }^{1}$. Recurrences are common.

UTI depends on age of patients, time and geographical location. UTI is common in both males and females. Females are more susceptible than males, with three quarter of UTI occurring in pregnant women and one quarter in non-pregnant women. It is the most common form of bacterial infection in women with $10 \%$ developing UTI every year.

Enterobacteriaceae species like Escherichia coli, Proteus mirabilis, Enterobacter aerogenes, Citrobacter freundii and Klebsiella pneumoniae are the commonest uropathogens implicated in bacteremia and nosocomial infections. These are the 
common organisms producing Extended Spectrum Beta-Lactamase ${ }^{2}$.

The aim of this study is to detect ESBL producing uropathogens among multi drug resistant pathogens causing UTI, since the treatment failure of UTI are commonly caused by Enterobacteriaceae. The prevalence of ESBLs in Puducherry is 67\% among Escherichia coli, 57\% among Klebsiella species and 55\% among Enterobacter species $^{3}$.

The main and objectives of this study, to identify and study the incidence of extended spectrum beta-lactamase producing Enterobacteriaceae in urinary tract infections by different phenotypic detection test.

\section{Materials and Methods}

About 100 Uropathogens isolated from urine samples of inpatients and outpatients of SVMCH during 2014-2015 were included.

\section{Inclusion Criteria}

Enterobacteriaceae group of organisms isolated from urine samples with significant bacteriuria.

\section{Exclusion Criteria}

Non enterobacteriacea group of bacterias and Insignificant bacteriuria.

A questionnaire and informed consent was obtained from all the patients before collecting sample. Institutional ethical committee clearance was obtained before the commencement of the study.

Urine samples are collected by the cleancatch technique. In women, the periurethral area and the perineum are first cleaned with 2 or 3 guaze pads soaked in soapy water, using a forward-to-back motion, followed by a rinse with saline or water. The midstream urine is then collected in a sterile, wide mouth container with a tightly fitted lid. In men, simply cleansing of the urethral meatus immediately before voiding and then collection of the midstream urine is usually sufficient.

The samples were processed without any delay. The samples were inoculated on blood agar, MacConkey agar and CLED agar and incubated at $37^{\circ} \mathrm{C}$ for $24 \mathrm{hrs}$. Based on the colony morphology, gram's staining and biochemical tests, GNB belonging to Enterobacteriaceae family were isolated and subjected to AST by Kirby-Bauer's disc diffusion method.

\section{ESBL Screening}

All the isolates showing resistance to third generation cephalosporins were further tested for confirmation of beta-lactamase production by phenotypic methods.

\section{Double Disc Synergy Test}

Organism suspension was adjusted to 0.5 McFarland's standard and was streaked onto MHA plate. Two different cephalosporin discs-cefotaxime, ceftriaxone were placed around the amoxicillin-clavulanate disc (Himedia) at a center to center distance of $30 \mathrm{~mm}$ (center to center). Enhancement of zone of inhibition of third generation cephalosporins caused by clavulanate present in amoxicillin-clavulanate disc was interpretated as ESBL producer. E. coli ATCC 25922 was used as the positive control $^{4}$.

Disc strength: $30 \mu \mathrm{g}$ disc, third generation

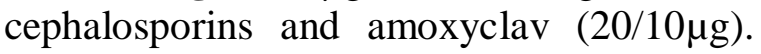
Cefpodoxime is the best sentinel antibiotic for finding resistance to third generation cephalosporins 


\section{Phenotypic Confirmatory Test}

Cefotaxime was used alone as well as in combination with clavulanic acid or any other beta-lactamase inhibitor which was placed $30 \mathrm{~mm}$ apart (center to center) on MHA and incubated at $37^{\circ} \mathrm{C}$ for 24 hrs. More than $5 \mathrm{~mm}$ increase in the zone of combination disc were phenotypically confirmed as ESBL producer.

The upper half of the above picture shows > $5 \mathrm{~mm}$ increase in the zone of inhibition of combination disc $(\mathrm{CEC}+\mathrm{CTX})$ indicating ESBL producer by PCT.

\section{E- Test}

It is useful for quantitative determination of susceptibility of bacteria to antibacterial agents. The system comprises of a predefined quantitative gradient which is used to determine the MIC in $\mathrm{mcg} / \mathrm{ml}$ of different antimicrobial agents against microorganisms as tested on appropriate agar media, following overnight incubation.

\section{Ezy MIC Strip Features and Advantages}

The strip is made up of porous paper material unlike plastic non-porous material.

The strip has MIC values printed on both sides identically.

The antimicrobial agent is evenly distributed on either side of the Ezy MIC strip and hence it can be placed by any side on the agar surface.

For Ezy MIC strips, MIC values can be read without opening the lid of the plate as most commonly translucent medium such as MHA is employed .

Once placed, the strip is adsorbed within 60 seconds and firmly adheres to the agar surface.

The strip does not form air bubbles underneath, and hence there is no need to press the strip.

Preparation of inoculum: From the culture plate, four to five colonies were taken with sterile bacteriological loop and inoculated into broth and incubated at $37^{\circ} \mathrm{C}$ for 2 to 8 hrs. The suspension turbidity was compared to 0.5 McFarland's standard.

\section{Method}

A sterile non-toxic swab was dipped into the standardized inoculum and rotated firmly against the upper inside wall of the tube to express excess fluid.

The entire agar surface was streaked with the swab three times, turning the plate at $60^{\circ}$ angle between each streaking.

The strip was placed at room temperature for 15 minutes before opening.

With the applicator, the strip was placed at a desired position on agar surface swabbed with test culture.

The plate was incubated at $37^{\circ} \mathrm{C}$ for $24 \mathrm{hrs}$.

The MIC was interpreted from the point where the ellipse intersects the MIC scale on the strip.

In the above picture, the ratio of CTX/ CTX+ is more than 8, which indicates ESBL positive strain.

\section{ESBL Chrom Agar}

This test is a rapid screening method for the detection of gram negative ESBL producing bacteria. It also inhibits the growth of those bacteria carrying AmpC type resistance and 
thus helps to avoid ESBL false positive test reading due to intrinsic $\mathrm{AmpC}$ resistance in the classical testing methods. It has high sensitivity and specificity.

Composition: Powder base (CHROM agar orientation). Supplement (CHROM agar ESBL supplement).

\section{Inoculation}

The samples are inoculated onto the plate by direct streaking or by spreading technique and incubated at $37^{\circ} \mathrm{C}$ for $18-24 \mathrm{hrs}$.

In the above picture, pink colour colonies represents ESBL E. coli and metallic blue colour colonies represents ESBL Klebsiella or Enterobacter or Citrobacter.

\section{Results and Discussion}

A total of 170 urine samples were received from in-patient and out-patient departments of our hospital. Out of which 140 samples showed significant growth. Totally, 100 were GNB belonging to Enterobacteriaceae family

This chart shows the age of the patients affected by UTI. Present study showed that $39 \%$ of the infected were above 50 years of age and $61 \%$ were below 50 years of age.

It was observed in our study that out of 100 patients studied, $33 \%$ were males and $67 \%$ were females.

Above data shows that among 100 patients used for the study, $63 \%$ of the patients were in-patients (hospital acquired infections) and $37 \%$ of the patients were out-patients (community acquired infections).

The above chart shows that, out of 100 patients used for the study, 32\% were catheterized and $68 \%$ were non-catheterised.

The above graph shows the percentage of the uropathogens causing the infection. In this study, the percentage of patients affected by various uropathogens namely $E$. Coli, Citrobacter, P. vulgaris, K. oxytoca, $P$. mirabilis, $K$. pneumoniae, Enterobacter aerogenes and Morganella morgagnii were found to be $52 \%, 4 \%, 5 \%, 6 \%, 13 \%, 16 \%$, $3 \%$ and $1 \%$ respectively. Among the uropathogens isolated, E. coli was found to be the most common.

The above table shows that among 52 isolates of E. coli $40 \%$ is ESBL producer, among 4 isolates of Citrobacter 50\% was ESBL producer, out of 5 isolates of $P$. vulgaris 20\% was ESBL producer, out of 6 isolates of $K$. oxytoca $33 \%$ was ESBL producer, out of 13 isolates of $P$. mirabilis $23 \%$ was ESBL producer, out of 16 isolates of $K$. pneumoniae $25 \%$ was ESBL producer, out of 3 isolates of E. aerogenes $67 \%$ was ESBL producer and none of the isolates of $M$. morgagnii was ESBL producers.

In this study, double disc synergy test was performed to test the presence of ESBL. Out of 100 isolates tested, $29 \%$ of the isolates showed positive results and $71 \%$ of the isolates showed negative results.

In this study, phenotypic confirmatory test was performed. Among 100 isolates tested, $35 \%$ isolates were ESBL positive and $65 \%$ were ESBL negative.

In this study, E-test was performed to test the zone of inhibition of ESBL producers. Among the 100 isolates tested, 35\% of the isolates were ESBL positive and the remaining $65 \%$ were ESBL negative In this study among 100 isolates tested, 35\% were positive for ESBL production by ESBL 
CHROM agar test and $65 \%$ were negative for ESBL production.

The above table shows that there is a significant difference in different methods used for ESBL detection such as double disc synergy test $\left(X^{2}=75.85, p=0.000\right)$, phenotypic confirmatory test $\left(X^{2}=100.00\right.$, $\mathrm{p}=0.000)$, ESBL CHROM agar test $\left(\mathrm{X}^{2}=\right.$ $100.00, \mathrm{p}=0.000)$ and $\mathrm{E}$-test $\left(\mathrm{X}^{2}=100.00, \mathrm{p}\right.$ $=0.000)$.
ESBL producing bacteria are increasing tremendously both in number and variety. Earlier, isolates were found to be more from hospital acquired infections, but recently reports of isolation from community acquired infections have become more common. The increase in resistance rate has made it necessary to identify the enzymes in the urine isolates.

Table.1 E-test Interpretation

\begin{tabular}{|c|c|c|}
\hline REPORT & FORMULA & INTERPRETATIVE CRITERIA \\
\hline ESBL positive & CTX/CTX $+>8$ & $\begin{array}{l}\text { When the ratio of the value obtained } \\
\text { for cefotaxime (CTX): the Value of } \\
\text { cefotaxime In combination with } \\
\text { clavulanic acid (CTX+) is more than } 8 \\
\text { or no zone is obtained for CTX and } \\
\text { zone obtained in CTX+ }\end{array}$ \\
\hline ESBL negative & $\mathrm{CTX} / \mathrm{CTX}+\leq 8$ & $\begin{array}{l}\text { When ratio of the value obtained for } \\
\text { cefotaxime (CTX): the value of } \\
\text { Cefotaxime in combination with } \\
\text { clavulanic acid }(\mathrm{CTX}+) \text { is less than } \\
\text { or equal to } 8\end{array}$ \\
\hline ESBL (non- conclusive) & & $\begin{array}{l}\text { When no zone of inhibition is } \\
\text { obtained on the either side In such } \\
\text { cases, resistance may be due to } \\
\text { mechanisms other than ESBL } \\
\text { production These have to be } \\
\text { investigated further before reporting. }\end{array}$ \\
\hline
\end{tabular}

Table.2 Chrom Agar Interpretation

\begin{tabular}{|c|c|}
\hline MICROORGANISM & TYPE OF COLONY \\
\hline ESBL E. coli & Dark pink to reddish \\
\hline ESBL Klebsiella, Enterobacter, Citrobacter & Metallic blue \\
\hline ESBL Proteus & Brown halo \\
\hline ESBL Acinetobacter & Cream \\
\hline ESBL Pseudomonas & Translucent \\
\hline Stenotrophomonas & Colourless \\
\hline Gram +ve strains & Inhibited \\
\hline
\end{tabular}


Table.3 Distribution of ESBL Producing GNB in Urine

\begin{tabular}{|c|c|c|c|c|c|c|c|c|}
\hline & \multicolumn{8}{|c|}{ BACTERIAL ISOLATES } \\
\hline & $\begin{array}{l}\dot{\tilde{\Xi}} \\
0 \\
\dot{0} \\
\\
(52 \%) \\
\end{array}$ & $(4 \%)$ & 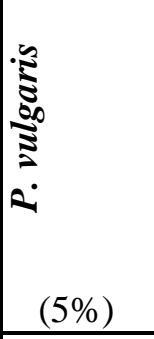 & 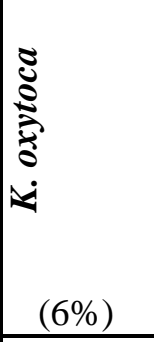 & 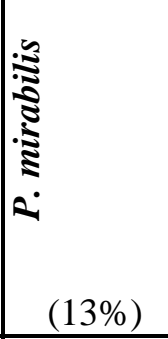 & 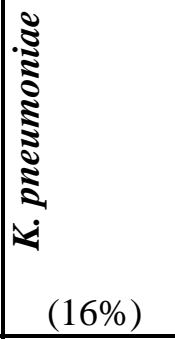 & 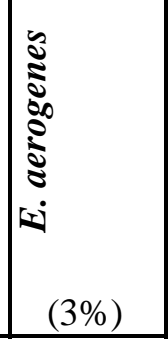 & 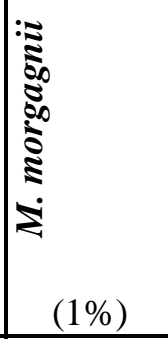 \\
\hline $\begin{array}{l}\text { ESBL } \\
\text { NON- } \\
\text { ESBL }\end{array}$ & $\begin{array}{l}21(40 \%) \\
20(38 \%)\end{array}$ & $\begin{array}{l}2(50 \%) \\
2(50 \%)\end{array}$ & $\begin{array}{l}1(20 \%) \\
4(80 \%)\end{array}$ & $\begin{array}{l}2(33 \%) \\
4(66 \%)\end{array}$ & $\begin{array}{r}3(23 \%) \\
10(77 \%)\end{array}$ & $\begin{array}{l}4(25 \%) \\
12(75 \%)\end{array}$ & $\begin{array}{l}2(67 \%) \\
1(33 \%) \\
\end{array}$ & $\begin{array}{c}0(0 \%) \\
1(100 \%)\end{array}$ \\
\hline
\end{tabular}

Table.4 Chi-Square Value and P-Value of DDST, PCT , Chrom Agar and E-Test

\begin{tabular}{|c|c|c|c|c|}
\hline $\begin{array}{l}\text { Phenotypic } \\
\text { Detection }\end{array}$ & ESBL & Non - SBL & $x^{2}$ & $\mathbf{P}$ \\
\hline $\begin{array}{l}\text { Positive } \\
\text { DDST } \\
\text { Negative }\end{array}$ & $\begin{array}{c}29(29 \%) \\
6(6 \%)\end{array}$ & $\begin{array}{c}0(0 \%) \\
65(65 \%)\end{array}$ & 75.85 & $0.000 * *$ \\
\hline $\begin{array}{c}\text { Positive PCT } \\
\text { Negative }\end{array}$ & $\begin{array}{c}35(35 \%) \\
0(0 \%)\end{array}$ & $\begin{array}{c}0(0 \%) \\
65(65 \%)\end{array}$ & 100.00 & $0.000 * *$ \\
\hline $\begin{array}{c}\text { Positive } \\
\text { CHROM agar } \\
\text { Negative }\end{array}$ & $\begin{array}{c}35(35 \%) \\
0(0 \%)\end{array}$ & $\begin{array}{c}0(0 \%) \\
65(65 \%)\end{array}$ & 100.00 & $0.000 * *$ \\
\hline $\begin{array}{l}\text { Positive E-test } \\
\text { Negative }\end{array}$ & $\begin{array}{c}35(35 \%) \\
0(0 \%)\end{array}$ & $\begin{array}{c}0(0 \%) \\
65(65 \%)\end{array}$ & 100.00 & $0.000^{* * *}$ \\
\hline
\end{tabular}

Plate.1 Double Disc Synergy Test

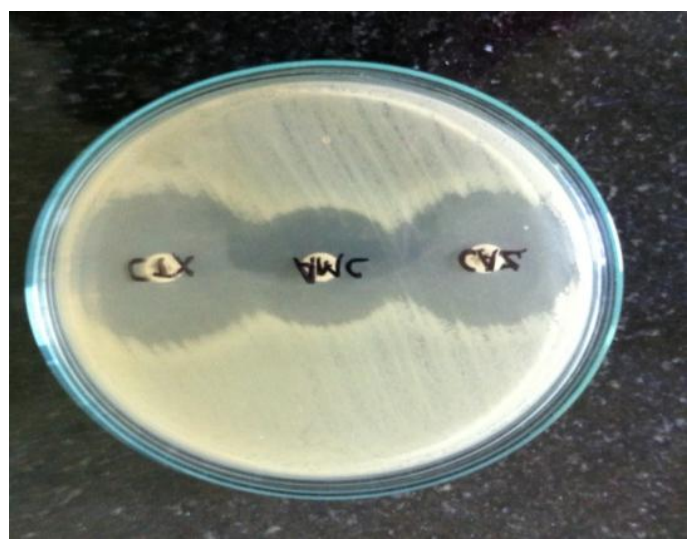


Plate.2 Phenotypic Confirmatory Test

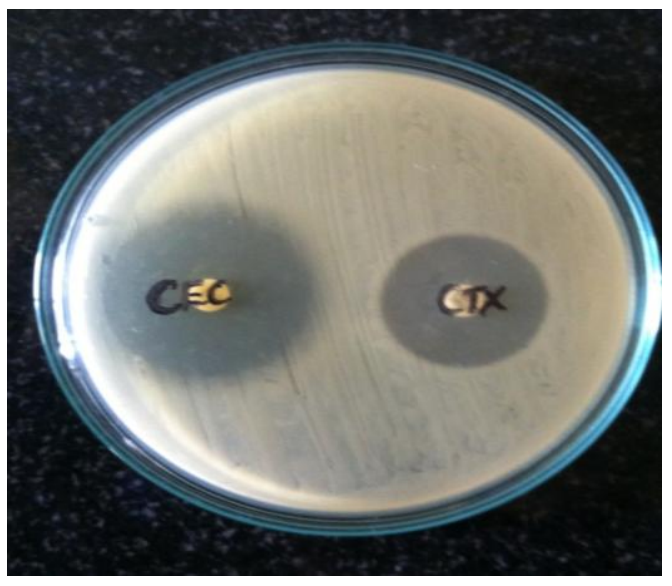

Plate.3 E-Test

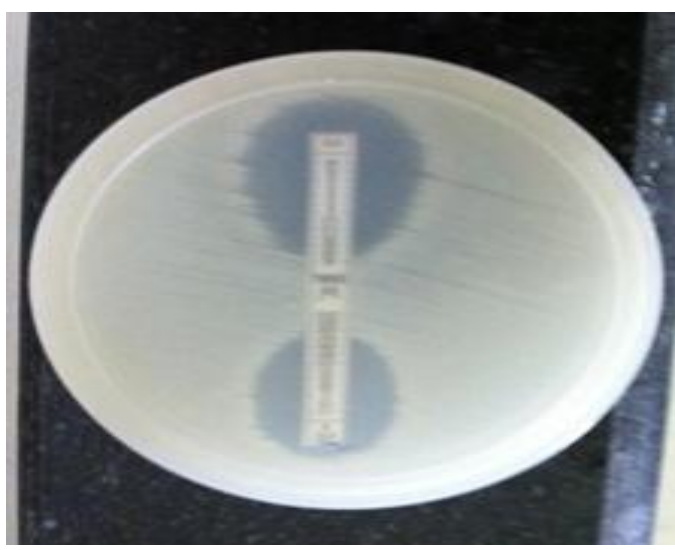

Plate.4 Chrom Agar

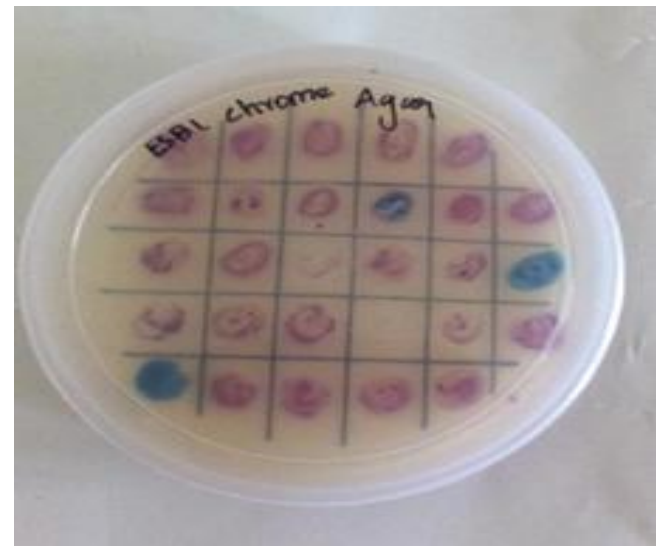


Chart.1 Age Wise Distribution of the Patients

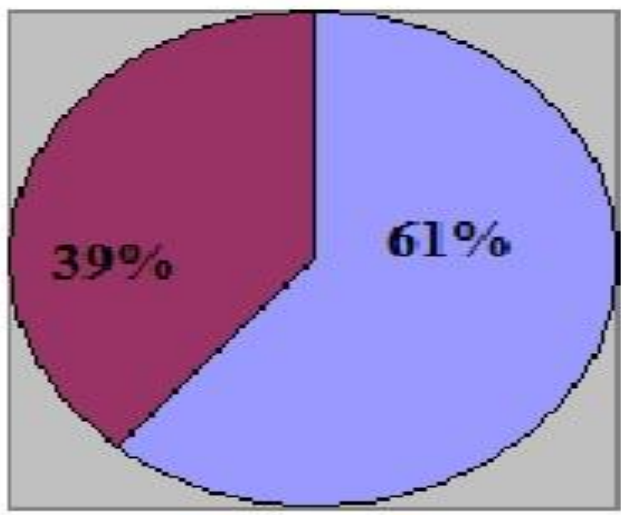

$\square<50$ YRS

$\square$ >5OYRS

Chart.2 Sex Wise Distribution of the Patients

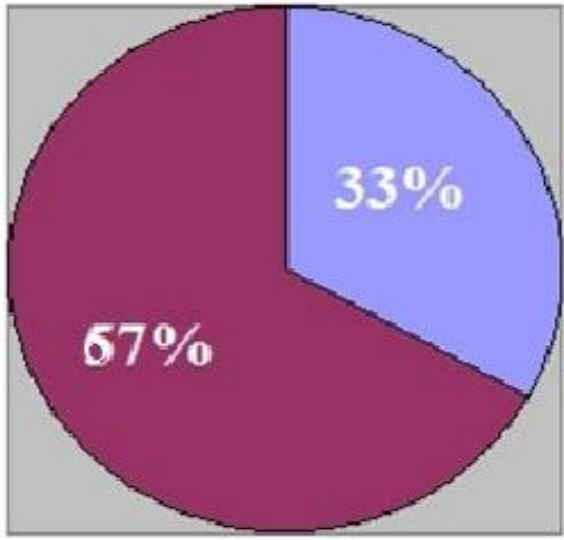

MALE

FEMALE

Chart.3 OP Versus IP Distribution of the Patients

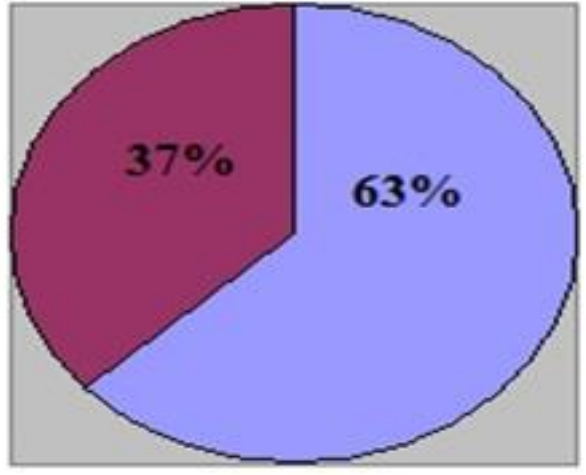

$\square$ INPATIENT

口 OUTPATIENT 
Chart.4 Catheterization

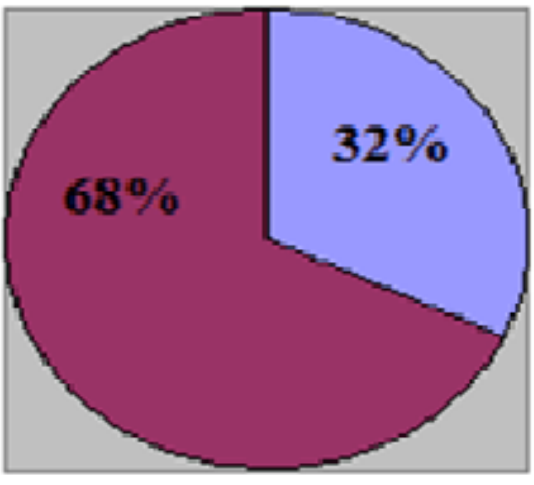

catheterised

anon-

catheterised

Chart.5 ESBL Detection by Double Disc Synergy Test

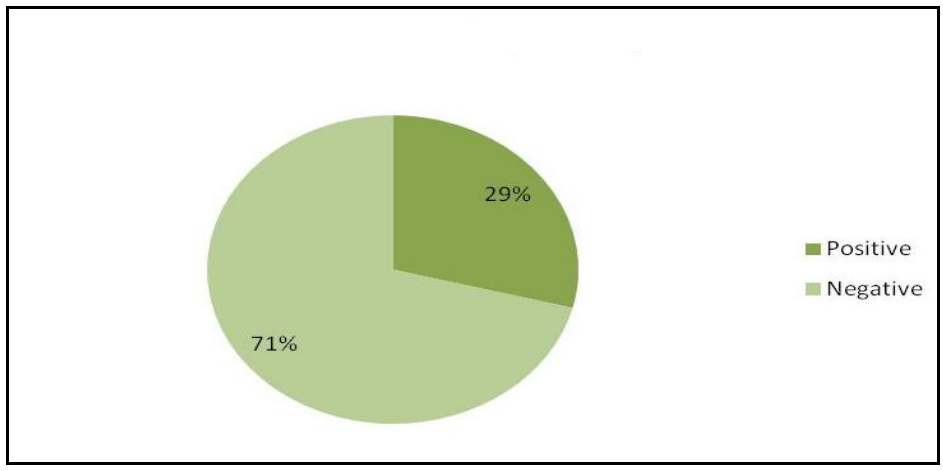

Chart.6 ESBL Detection by Phenotypic Confirmatory Test

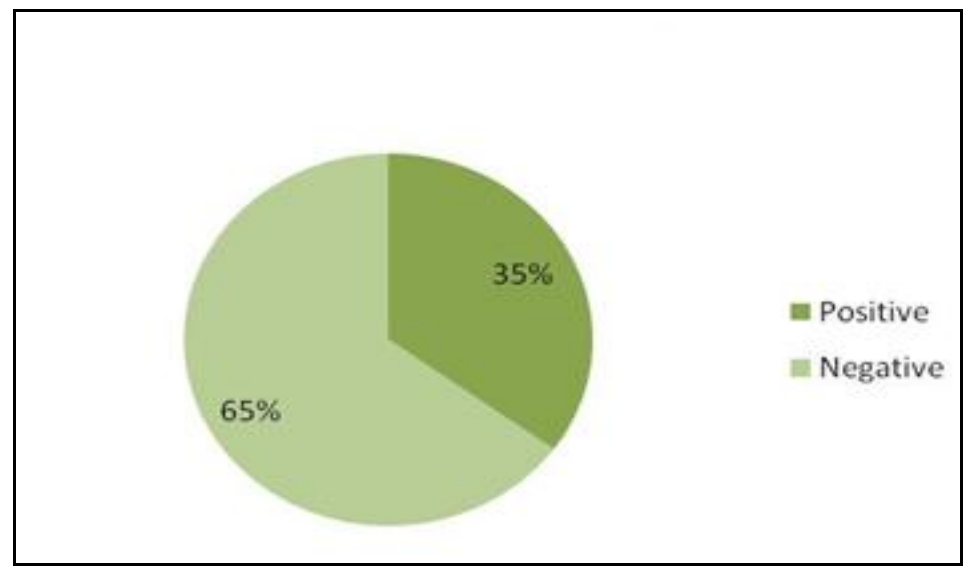


Chart.7 ESBL Detection by E-Test

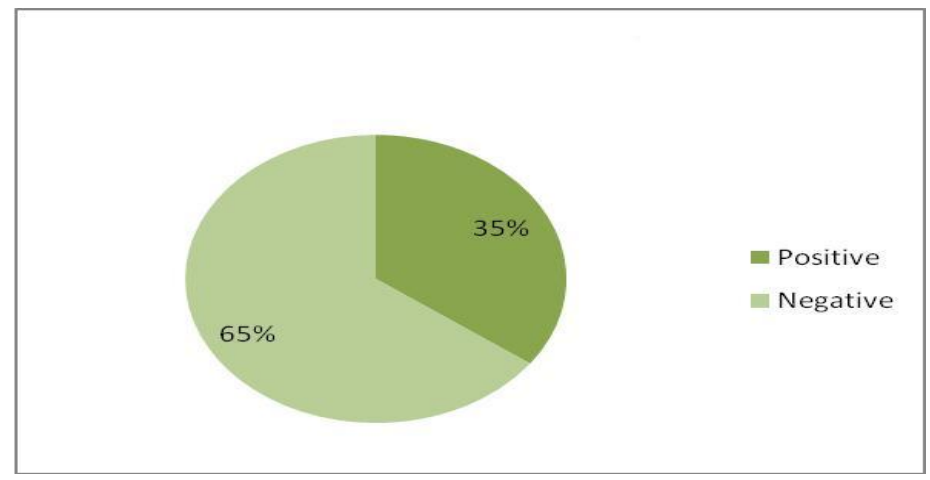

Chart.8 Detection of ESBL by Chrom Agar

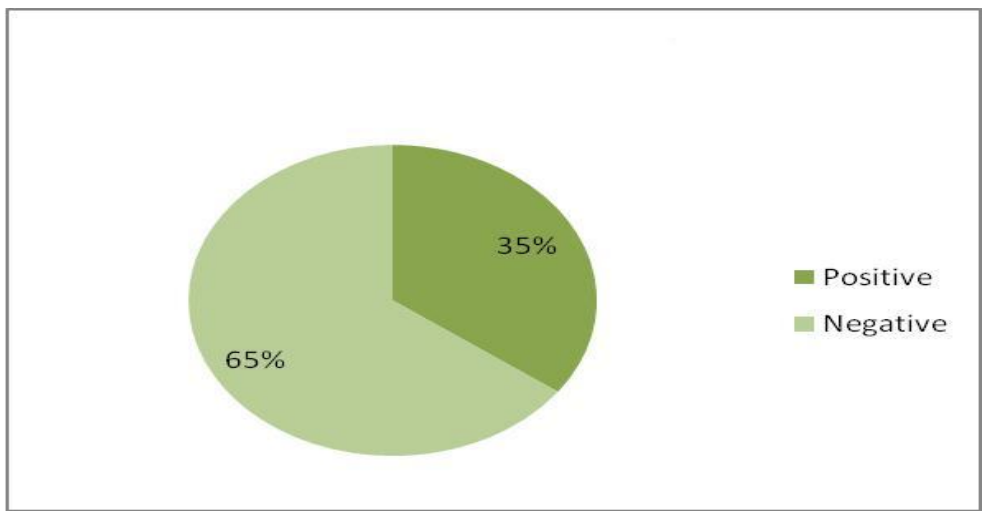

Graph.1 Distribution of GNB in Urine

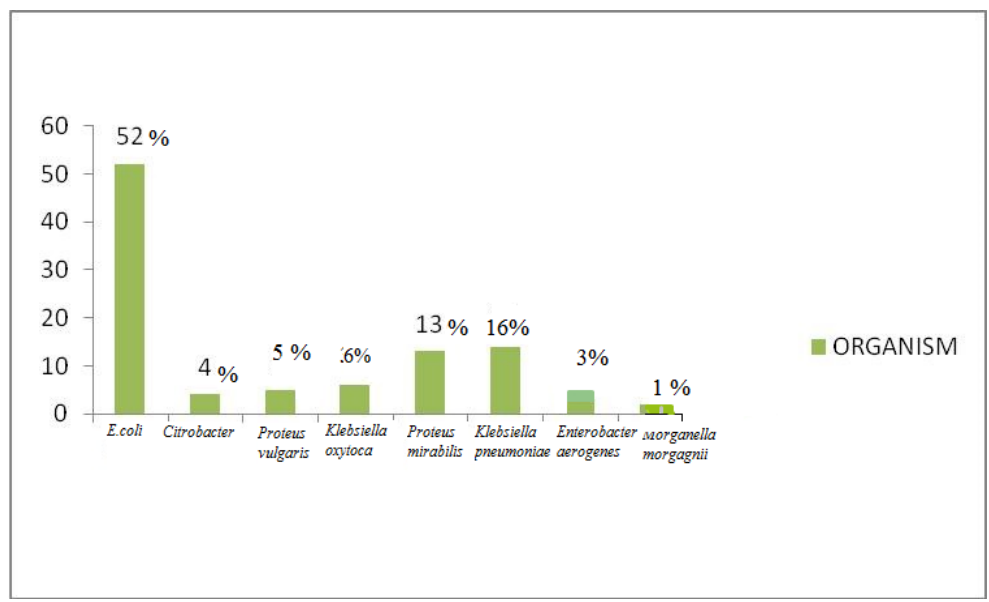

Contrary to the study done by Mahesh E $e t$ $a l^{5}$ which reported UTI prevalence to be more in the elderly people (53\%), in our study ESBL producers were predominantly isolated from patients below 50 years $(61 \%)$. In our study uropathogens were isolated more from women $(67 \%)$ than men $(33 \%)$, principally due to anatomic and 
physiological factors which is similar to the study done by Muvunyi et $a l^{6}$ where $71.9 \%$ of women were reported to have UTI and Moyo et $a l^{7}$ where $54.4 \%$ of females and $45.6 \%$ of males were reported to have UTI and Gurjeet Singh et $a l^{8}$ where $37 \%$ of males and $63 \%$ of females were affected and mean age is less than 50 years.

In our study ESBL producers were isolated more from inpatients, which is contrary to the study done by Dawoodabadi ${ }^{9}$ on urinary E. coli isolates where $68.18 \%$ was isolated from community and $31.82 \%$ was isolated from inpatients.

In our study $E$. coli remains the dominant pathogen with $10 \%$ isolates in community acquired UTI and 25\% isolates in hospital acquired infections, which is similar to the study done by Vandana berry et $a l^{10}$, where $24.75 \%$ isolates of E. coli accounts for community acquired UTI and 33\% isolates accounts for hospital acquired infections.

In a study done by Luca Arnaldo et $a l^{11}$ $51.8 \%$ of catheterized patients were found to be ESBL producers but in our study only $12 \%$ of catheterized patients were found to be ESBL producers.

Comparison methods employed for detection of ESBLs in our study showed DDST (29\%) was less sensitive than PCT method $(35 \%)$. NCCLS guidelines also state that PCT is more effective in detecting the ESBLs than DDST.

E test in our study showed $100 \%$ sensitivity. This is in correlation with the study of Ritu aggarwal et $a l^{12}$. In a study done by Enas Khater et $a l^{13}$, out of $45 \mathrm{E}$. coli strains, 24 $(53.3 \%)$ ESBL producers were detected by E-test (gold standard method for confirmation of ESBL according to CLSI) and $21(46.7 \%)$ strains were reported as non-
ESBL producers. In our study E-test detected $35 \%$ of ESBL producers, which is less compared to the study done by Enas Khater et al.

Panagea et $a l^{14}$ and Nordmann et $a l^{15}$ reported that CHROM agar is $100 \%$ sensitive in detecting ESBL producers. In our study CHROM agar detected 35\% ESBL producers.

The co-existence of both ESBL and AmpC beta-lactamase in some GNB has also been reported recently. The reason could be due to dissemination of plasmid mediated AmpC beta-lactamase among Enterobacteriaceae. There are more chances of getting false negative tests in ESBL detection by these strains. In our study, all ESBL producers were positive for AmpC production which is contrary to the study done by Varsha et $a l^{16}$, where only $8 \%$ were AmpC positive out of $52 \%$ of ESBL producers.

In studies done by Karou et $a l^{17}$ and Mayo et $a l$, both E. coli and Klebsiella were shown as the dominant uropathogens. In the present study, 52 of the 100 isolates were found to be $E$. coli, of which 21 isolates were ESBL producers which is similar to the studies conducted by Gupta et $a l^{18}$., 1999 and Tambekar et $a l^{19}$., 2006, which have revealed $E$. coli to be one of the most potent uropathogen. But in a study done by Ritu agarwal et al, Klebsiella was found to be the major uropathogen which is contrary to our study.

ESBL strains are responsible for the spread of resistant genes, early detection of these strains is of great significance. The higher incidence, $58 \%$ ESBL production amongst GNB isolates were reported by other workers, however, the incidence of $35 \%$ ESBL production in our study was not in 
accordance to the studies reported by other investigators.

DDST was less effective than PCT, E- test and CHROM agar. PCT, E-test and CHROM agar were equally effective in detecting ESBL production. E-test and CHROM agar being costly, PCT can be used for detection of ESBL in routine lab setting.

In conclusion, ESBL producing organisms accounts for high morbidity and mortality. Hence for early detection of those ESBL producing isolates, ESBL detection methods like DDST, PCT can be performed in microbiology laboratories as routine test, as they are simple to perform and cost effective. Those isolates which are found to be ESBL producers by screening method can be confirmed by tests like E-test, ESBL CHROM agar which are found to have to be equally effective in detecting ESBL producers.

\section{References}

1. Miller LG, Tang AW. Treatment of uncomplicated urinary tract infections in an era of increasing antimicrobial resistance. Mayo Clin Proc. 2004;79:1048-53.

2. Grude N, Tveten Y, Jenkins A, Kristiansen BE.Uncomplicated urinary tract infections.Bacterial findings and efficacy of empirical antibacterial treatment.Scand J Prim Health Care. 2005 Jun;23:115-9.

3. Manobalan K, Menezes GA, Harish BN. Study of extended spectrum betalactamases and AmpC in Escherichia coli, Klebsiellaspp and Enterobacterspp in a tertiary care hospital. Res. J. Pharm. Biol. Chem. Sci. 2014 Dec;5(6):184189.

4. Foxman B. Epidemiology of urinary tract infections: incidence, morbidity, and economic costs. Am J Med. 2002;113(Suppl 1)A:5S - 13S.

5. Mahesh E, Medha Y, Indumathi VA, Prithvi SK, Mohammed WK, Punith K et al. Community-acquired urinary tract infection in elderly. BJMP 2011;4:40624.

6. Muvunyi CM, Masaisa F, Bayingana C, Mutesa L, MusemakweriA. Decreased susceptibility to commonly used antimicrobial agents in bacterial pathogens isolated from urinary tract infection in Rwanda: Need for new antimicrobial guidelines. Am. J. Trop. Med.Hyg.2011;84:923-28.

7. Moyo SJ, Aboud S, Kasubi M, Lyamuya EF, Maselle SY. Antimicrobial resistance among producers and non-producers of extended spectrum beta-lactamases in urinary isolates at a tertiary hospital in Tanzania. BMC Res Notes. 2010;3:348.

8. Gurjeet S, Raksha, Urhekar AD. Urinary tract infections: Prevalence and antimicrobial susceptibility pattern. Int $\mathbf{J}$ CurrMicrobiolAppl Sci. 2013;2:188-91.

9. Davoodabadi A, Farahani A, Ranjbaran M. "Antibiotic resistance in Escherichia coli strains isolated from urine of inpatients and outpatients". ZahedanJ. Res Med Sci. 2012;14:95.

10. Vandana B, Madan L, Vidya S, Rajesh $S$. Prevalence and antibiogram of gram negative bacilli with special reference to extended spectrum beta-lactamase production.JEvo Med Den Sci. 2012;1:916-20.

11. Lucas Arnold, Baquero F, Perez-Diaz JC, Martinez JL. Factors determining resistance to $\beta$-lactam combined with $\beta$ lactamase inhibitor in Eschericia coli. $\mathrm{J}$ Antimicrob Chemother.1991; 27: 569575

12. Ritu A, Uma C, Rama SA, Rohtak. Detection of extended spectrum betalactamase production among 
uropathogens. J. Lab Physic. 2009;1:710.

13. Khater E, Hammouda WS. Rapid detection of extended spectrum $\beta$ lactamase (ESBL) producing strain of Escherichia coli in urinary tract infection patients in Benha University Hospital, Egypt.J. British. Microbiol. Res. 2014;4:443-53.

14. Panagea T, Galani I, Souli M. Evaluation of CHROMagarTM KPC for the detection of carbapenemase producing Enterobacteriaceae in rectal surveillance cultures. Int $\mathrm{J}$ Antimicrob Agents. 2011; 37: 124-8

15. Nordmann P, Poirel L, Carrer A, Toleman MA, Walsh TR. How to detect NDM-1 producers. J. Clin. Microbiol. 2011;49:718-721.

16. Varsha G, Hena R, Nidhi S, Neelam K, Jagdish C. Determination of extended spectrum beta-lactamases and AmpC production in uropathogeic isolates of E.coli and susceptibility to fosfomycin. J Lab Physicians. 2009;5:90-3.

17. Karou, SD, Ilboudo DP, Nadembega WMC, Ameyapoh Y, Ouermi D. Antibiotic resistance in urinary tract bacteria in ouagadougou. Pak. J. Biol. Sci. 2009;12:712-16.

18. Gupta E, Mohanty S, Sood S, Dhawan B, Das BK, Kapil A et al. Emerging resistance to carbapenems in a tertiary care hospital in north India. Indian J Med Res. 2006;124:95-8.

19. Tambekar DH, Dhanorkar DV, Gulhane SR, Khandelwal VK, Dudhane MN. Antibacterial susceptibility of some urinary tract pathogens to commonly used antibiotics. Afr J Biotechnol. 2006;5:1562-65.

\section{How to cite this article:}

Prabha, R., Joshy M. Easow and Swapna, M. 2016. Phenotypic detection of Extended Spectrum Beta- Lactamase producing uropathogens using DDST, PCT, Chrom agar and E-test - A comparitive study. Int.J.Curr.Microbiol.App.Sci. 5(4): 565-577. doi: http://dx.doi.org/10.20546/ijcmas.2016.504.064 